\title{
A NEW APPROACH TO PROGRAM TESTING
}

James C. King, IBM T. J. Watson Research Center, Yorktown Heights, New York, USA

\begin{abstract}
The current approach for testing a program is, in principle, quite primitive. Some small sample of the data that a program is expected to handle is presented to the program. If the program produces correct results for the sample, it is assumed to be correct. Much current work focuses on the question of how to choose this sample. We propose that a program can be more effectively tested by executing it "symbolically". Instead of supplying specific constants as input values to a program being tested, one supplies symbols. The normal computational definitions for the basic operations performed by a program can be expanded to accept symbolic inputs and produce symbolic formulae as output.
\end{abstract}

If the flow of control in the program is completely independent of its input parameters, then all output values can be symbolically computed as formulae over the symbolic inputs and examined for correctness. When the control flow of the program is input dependent, a case analysis can be performed producing output formulae for each class of inputs determined by the control flow dependencies. Using these ideas, we have deșigned and implemented an interactive debugging/testing system called EFFIGY.

\section{INTRODUCTION}

As tools for tealizing correct programs, program testing and program proving are the ends of a spectrum whose range is the number of times the program must be executed. To establish its correctness through testing, one must execute the program at least once for all possible unique inputs; usually an infinite number of times. To establish its correctness through a rigorous correctness proof, one need not execute the program at all; but he may be faced with a tedious, if not difficult, formal analysis. These two extreme points of the spectrum offer other contrasts as well. Correctness proofs usually ignore certain realities encountered in actual test runs, for example, machine dependent details like overflow and precision. (One notable effort to bring machine dependent issues into correstness proofs is the recent thesis by Sites [7]). On the other hand one may finish a proof of correctness, but seldom do we ever finish testing a program. Normal testing and correctness proofs also differ in the degree to which the user is required to supply a formal specification of "correct" program behavior. While a careful statement of correctness may be recommended for program testing, it is not required. A user 
may choose an interesting input case and then decide a posteriori, in this specific case, if the output appears to be correct. In a formal proof of correctness one must have a careful program specification.

A testing tool is described in this paper which allows one to choose intermediate points on the spectrum between individual test runs and general correctness proofs. One can perform a single "symbolic execution" of the program that is equivalent to a large (usually infinite) number of normal test runs. Test run results can not only be checked by careful manual inspection but if a machine interpretable program specification is supplied with the program it can be used to automatically check the results. Furthermore, by varying the degree to which symbolic information is introduced into the symbolic execution one can move from normal execution (no symbolic data) to a symbolic execution which, in some cases, provides a proof of correctness.

\section{SYMBOLIC EXECUTION}

The notion of symbolically executing a program follows quite naturally from normal program execution. First assume that there is a given programming language and a normal definition of program execution for that language. This execution definition must be used for production executions but an alternative symbolic execution semantics for the language can be defined to great advantage for debugging and testing. The individual programs themselves are not to be altered for testing. The definition of the symbolic execution must be such that trivial cases involving no symbols should be equivalent to normal executions and any information learned in a symbolic execution should apply to the corresponding normal executions as well.

An execution of a procedure becomes symbolic by introducing symbols as input values in place of real data objects (e.g., in place of integers and floating point numbers). Here "inputs" is to be taken generally meaning any data external to the procedure, including that obtained through parameters, global variables, explicit READ statements, etc. Choosing symbols to represent procedure inputs should not be confused with the similar notion of using symbolic program variable names. A program variable may have many different specific values associated with it during a particular execution whereas a symbolic input symbol is used in the static mathematical sense to represent some unknown yet fixed value. Values of program variables may be symbols representing the non-specific procedure inputs.

Once' a procedure has been initiated and given symbolic inputs, execution can proceed as in a normal execution except when the symbolic inputs are encountered. This occurs in two basic ways: computation of an expression involving procedure inputs, and conditional branching dependent on procedure inputs.

\section{Computation of Expressions}

The programming language has a set of basic computational operators such as addition $(+)$, multiplication $\left(^{*}\right)$, etc. which are defined over data objects such as integers. Each operator must be extended to 
deal with symbolic data. For arithmetic data this can be done by making use of the usual relationship between arithmetic and algebra. The arithmetic computations specified by these operators can be "delayed" or generaiized by the appropriate algebraic formula manipulations. For example, suppose the symbolic inputs $\alpha$ and $\beta$ are supplied as argument values to a procedure with formal parameter variables $A$ and $B$. Denote the value of a program variable $X$ by $v(X)$. Then initially, $v(A)=\alpha$ and $\mathrm{v}(\mathrm{B})=\beta$. If the assignment statement $\mathrm{C}:=\mathrm{A}+2^{*} \mathrm{~B}$ were symbolically executed in this context $\mathrm{C}$ would be assigned the symbolic formula $(\alpha+2 * \beta)$. The statement $D:=C-A$, if executed next, would result in $v(D)=2 * \beta$.

Similar symbolic generalization can be done, at least in theory, for all computational operations in the programming language. In the most difficult case, one could at least record in some compact notation the sequence of computations which would have taken place had the arguments been non-symbolic. The success in doing this in practice depends upon how easily these recordings can be read and understood and how easily they can be subsequently manipulated and analyzed mechanically.

\section{Conditional Branching}

Consider the typical decision-making program statement, the $I F$ statement, taking the form:

IF $B$ THEN $S_{1}$ ELSE $s_{2}$,

where $B$ is some Boolean valued expression in the language and $S_{1}$ and $S_{2}$ are other statements. Normally, either $\mathrm{v}(B)=$ true and statement $S_{1}$ is executed or $\mathrm{v}(B)=$ false and statement $S_{2}$ is executed. However, during a symbolic execution $\mathrm{v}(B)$ could be true, false or some symbolic formula over the input symbols. Consider the latter case. The predicates $v(B)$ and $-v(B)$ represent complementary constraints on the input symbols that determine alternative control flow paths through the procedure. For now, this case is called an "unresolved" execution of a conditional statement. The notion will be refined as the presentation develops. Since both alternatives paths are possible the only complete approach is to explore both: the execution forks into two "parallel" executions, one assuming $v(B)$, the other assuming $\rightarrow \mathrm{v}(B)$.

Assume the execution has forked at an unresolved conditional statement and consider the further execution for the case where $v(B)$. The execution may arrive at another unresolved conditional statement execution with associated boolean, say $C$. Expressions $\mathrm{v}(B)$ and $\mathrm{v}(C)$ are both over the procedure input symbols and it is possible that either $\mathrm{v}(B) \supset \mathrm{v}(C)$ or $\mathrm{v}(B) \supset \neg \mathrm{v}(C)$. Either implication being true would show that the assumption made at the first unresolved execution, namely $v(B)$, is strong enough to resolve the subsequent test, namely to show that either $v(C)$ or $\neg v(C)$.

Because the assumptions made in the case analysis of one unresolved conditional statement execution may be effective in resolving subsequent unresolved statement executions they are preserved as part of the execution state, along with the variable values and the statement counter, and are called the "path condition" (denoted pe). At the beginning of a program execution the pe is set to true. The revised 
rule for symbolically executing a condition statement with associated Boolean expression $B$ is to first form $\mathrm{v}(B)$ as before and then form the expressions:

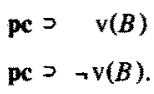

If pc is not identically false then at most one of the above expressions is true. If the first is true the assumptions already made about the procedure inputs are sufficient to completely resolve this test and the execution follows only the $\mathrm{v}(B)$ case. Similarly if the second expression is true it follows the $\neg \mathrm{v}(B)$ case. Both of these cases are considered "resolved" or non-forking executions of the conditional statement.

The remaining case when neither expression is true is truly an unresolved (forking) execution of the conditional statement. Even given the earlier constraints on the procedure inputs $(\mathbf{p c}), \mathrm{v}(B)$ and $\neg \mathrm{v}(B)$ are both satisfiable by some non-symbolic procedure inputs. As discussed above, unresolved conditional statement executions fork into two parallel executions. One when $v(B)$ is assumed, in which case the pc is revised to pe $A \mathrm{v}(B)$, the other when $\neg \mathrm{v}(B)$ is assumed and then pc becomes pc $A \neg \mathrm{v}(B)$. Note that the forking is a property of a conditional statement execution not the statement itself. One execution of a particular statement may be resolved yet a later execution of the same statement may not.

The pe is the accumulator of conditions on the original procedure inputs which determine a unique control path through the program. Each path, as forks are made, has its own pe. No pe is ever identically false since the original pe is true and the only changes are of the form $\mathbf{p c}:=\mathbf{p c} \wedge \mathrm{q}$ and those only in the case when $p c \wedge q$ is satisfiable $((p c \wedge q)=\neg(p c \supset \neg q)$ which is satisfiable if $p c \supset \neg q$ is not a theorem). Each path caused by forking also has a unique pe since none are identically false and they all differ in some term, one containing a $q$ the other $a \neg q$.

\section{SYMBOLIC EXECUTION TREE}

One can characterize the symbolic execution of a procedure by an "execution tree". Associate with each program statement execution a node and with each transition between statements a directed arc connecting the associated statement nodes. For each forking (unresolved) conditional statement the associated execution node has more than one arc leaving it labeled by and corresponding to the path choices made in the statement. In the previous discussion of IF statements there were two choices corresponding to $\mathrm{v}(B)$ and $\neg \mathrm{v}(B)$. The node associated with the first statement of the procedure would have no incoming arcs and the terminal statement of the procedure (RETURN or END statement) is represented by a node with no outgoing arcs.

Also associate the complete current execution state, i.e., variable values, statement counter, and pc with each node. In particular, each terminal node will have a set of program variable values given as formulae over the procedure input symbols, and a pe which is a set of constraints over the input 
symbols characterizing the conditions under which those variable values would be computed. $A$ user can examine these symbolic results for correctness as he would normal test output or substitute them into a formal output specification which should then simplify to true.

The execution tree for a program will be infinite whenever the program contains a loop for which the number of interations is dependent, even indirectly, on some procedure inputs. It is this fact that prevents symbolic execution from directly providing a proof of correctness technique. Symbolic execution is indeed an execution and at least in this simplest form described here provides an advanced testing methodology. Burstall [1] has independently developed the notion of symbolic execution and added the required induction step needed to have a complete proof of correctness method. Dentsch [2], also independently, developed the notion of symbolic execution as an implementation technique for an interactive program prover based on Floyd's method [3]. In fact, one can see the basic elements of the notion of using symbolic execution as the basis for a correctness method in the earlier work of Good [4]. The author and his colleagues have been pursuing the idea of symbolic execution in its own right as a debugging/testing technique. A particular system we have built called EFFIGY is described briefly in the next section.

\section{EFFIGY - AN INTERACTIVE SYMBOLIC EXECUTOR}

The author and his colleagues at IBM Research have been developing an interactive symbolic execution system for testing and debugging programs written in a simple PL/I style programming language. The language is restricted to integer valued variables and vectors (one dimensional arrays), It has many interactive debugging features including: execution tracing, break-points, and state saving/restoring. Of course, it provides symbolic execution and uses a formula manipulation package and theorem prover developed previously by the author $[5,6]$.

The general facilities and capabilities avallable are all that is of real interest and these are perhaps simplest and most economically explained by a system demonstration. An APPENDIX is included which shows an actual script (annotated in italics) from such a demonstration. A method for exploring execution trees with their multitude of forks and parallel executions is up to the user. He is provided the ability to choose particular forks at unresolved conditional statement executions (via go true, gofalse, and assume) and also has the state save/restore ability so that he may return to unexplored alternatives later. We are currently experimenting with various "test path-managers" which would embody some heuristics for automating this process, exhaustively exploring all the "interesting" paths. As with previous testing methods the crucial issue is: if one cannot execute all cases, which ones should he do; which are the interesting ones.

We are also working on practical methods for dealing with more advanced programming language 
features such as pointer variables. While, as mentioned above, most such enhancements are straightforward "in theory" many offer fundamental problems in practice.

\section{CONCLUSION}

Interactive debugging/testing systems have shown themselves to be powerful, useful tools for program development. A symbolic execution capability added to such a system is a major improvement. The normal facilities are always available as a special case. In addition, the basic system components of a symbolic executor provide a convenient toolbox for other forms of program analysis, including program proving, test case generation, and program optimization. Since such a system does offer a natural growth from today's systems, an evolutionary approach for achieving the systems of tommorrow is available. Valuable user experience and support is also provided. While practical use of the EFFIGY system is still quite limited, considerable insight into and understanding of the general notion of symbolic execution has been gained during its construction.

\section{ACKNOWLEDGMENTS}

The colleagues at IBM Research collaborating with me in this work are: S. M. Chase, A. C. Chibib, J. A. Darringer, and S. L. Hantler. They have all contributed significantly to the ideas presented here and to the design and implementation of our EFFIGY system. We also appreciate the support and encouragement received from D. P. Rozenberg, P. C. Goldberg, and P. S. Dauber. The manuscript was typed by J. M. Hanisch.

\section{REFERENCES}

[1] Burstall, R. M. Program proving as hand simulation with a little induction, IFIP Congress 74 Proc., Aug. 1974, pp. 308-312.

[2] Deutsch, L.P. An interactive program verifier, Ph.D. dissertation, Dept. Comp. Sci., Univ. of Calif,, Berkeley CA., May 1973.

[3] Floyd, R.W. Assigning meanings to programs, Proc. Symp. Appl. Math., Amer. Math. Soc., vol. 19, pp. 19-32, 1967.

[4] Good, D.I. Toward a man-machine system for proving program correctness, Ph.D. dissertation, Comp. Sci. Dept., Univ. of Wisc., Madison, Wisc., June 1970.

[5] King, J.C. and Floyd, R.W. An interpretation oriented theorem prover over integers, Journal of Comp. and Sys. Sci., vol. 6, no. 4, August 1972, pp. 305-323.

[6] King, J.C. A program verifier, IFIP Congress 71 Proc., Aug. 1971, pp. 235-249.

[7] Sites, R.L. Proving that computer programs terminate cleanly, Ph.D. dissertation, Comp. Sci. Dept., Stanford Univ., Stanford, CA., May 1974. 


\section{APPENDIX}

A seript from an actual EFFIGY session is shown below. The user's inputs are in lowercase letters and the system responses are in uppercase letters. To prevent any possible confusion the symbol " shown here to the left of the user inputs. Explanatory comments, in italic letters, have been added as a right hand column.

When EFFIGY is initially invoked it is in an "immediate" mode and will execute statements as they are typed. Any statement executed in this context is considered part of a main initial procedure called MAIN. The concept of the MAIN procedure and the concept of immediate execution are distinct since statements can also be executed in an immediate mode in the context of other procedures. MAIN is unique in that it has an immediate mode only and it is the only procedure privileged to execute the managerial system commands. Programs are made available to EFFIGY for stored program execution by declaring them, in MAIN, with the PROC statement similar to the way that internal procedures are declared in PL/1. However, EFFIGY does consider all procedures as EXTERNAL and they must be declared in MAIN.

Procedures are tested by a CALL from MAIN. Symbolic inputs can be supplied by enclosing a symbol string in double quotes, e.g., "a", "Dog". These symbolic constants can be used in most places instead of integer constants. The system responses drop the quotes since the context always makes the distinctions between different uses of identifiers quite clear. Values always involve the input symbols and never program variable names. Formulae are stored internal to EFFIGY in a "normalized" form and some of the expressions may appear quite different from what one might expect (e.g., $A<0$ will be typed out as $A \neg>-1$ ). The formulae are also kept in a simplified form (e.g., $2 * B=4$ is stored as $B-2=0$ ).

EFFIGY runs on CMS under VM/370 on an IBM/370 model 168. The CMS filing system and context editor are used as an integral part of EFFIGY for creating, changing, and storing procedures and command files. The INPUT command directs EFFIGY to read its input from the designated file (files have two part names in CMS) instead of directly from the user's terminal. As procedures are entered into EFFIGY (by a PROC ... END declaration) the statements are sequentially numbered. These statement numbers are used to reference particular points in the procedure for inserting breakpoints, turning tracing on and off, etc.

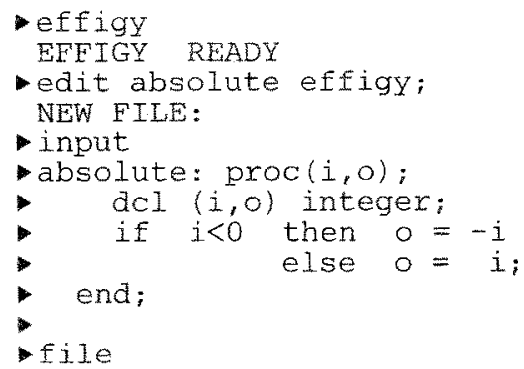

\author{
Inwoke the EFFIGY system. \\ Invoke,the CMS file editor and \\ type-in a new file called \\ 'absolute effigy'. \\ (end of input signified by null line.) \\ Save file permanently \&
}


- input absolute effigy;

1: ABSOLUTE: $\operatorname{PROC}(I, O)$;

2: DCL $(I, O)$ INTEGER;

3: IF $I<0$ THEN $O=-I$

4: $\quad$ ELSE $O=I$;

5: END;

- dal $z$ integer;

- call absolute $(55, z)$; display z; 55

- call absolute $(-66, z)$; display z; 66

- in absolute; turn all on all;

- in main;

-call absolute ("a",z);

1: ABSOLUTE: PROC $(I, O)$;

2: DCL (I,O) INTEGER;

3: IF $I<0$ THEN $O=-I$ $((A \neg>-1))$

TYPE GO TRUE OR GO FALSE

- before 5;

- savestate; STATE 1 SAVED

-go true;

$((A \rightarrow>-1))$

TRUE BRANCH

$\mathrm{O}=-\mathrm{A}$

STOPPED BETWEEN 3 AND 5

- display variables, assumption; IN ABSOLUTE

$I=A$

$\mathrm{O}=-\mathrm{A}$

$((A \neg>-1))$

-restore 1;

STATE 1 RESTORED. IN ABSOLUTE

- go false;

$((A \neg>-1))$

FALSE BRANCH

4: $\operatorname{ELSE} O=I$;

$\mathrm{O}=\mathrm{A}$

STOPPED BETWEEN

- display variables, assumption; IN ABSOLUTE

$I=A$

$\mathrm{O}=\mathrm{A}$

$((A \neg<0))$

$\rightarrow \mathrm{xgO}$;

BACK FROM ABSOLUTE TO MAIN

- display z;

A

- in absolute; turn all off all;

- in main;

- erase assumption;

-call absolute("a" - "b",z); go

TYPE GO TRUE OR GO FALSE $-A+B$

- edit absolute effigy;

next

- change /absolute/newabs/

- bottom

-up 1 go back to EFFIGY.

Have EFFIGY read input from that file.

Statements are numbered by EFFIGY.

Last line of file -- back to

terminal input.

Declare a variable in MAIN.

Try a numeric execution.

Result of display statement.

All tracing on in proc. 'absolute'.

Set back to MAIN.

Try a symbolic input " $a$ ".

Each statement execution is traced by printing $i t$.

Evaluated result of $I<0--v(B)$.

$(A \leq-1$ or $A<0)$

Unresolved (forking) IF-User option.

Stop before executing statement 5 .

Save the current execution state.

EFFIGY calls this state 1 .

Follow case where $A<0$.

$v(I<0)$-evaluated test.

Result of assignment to $O$.

Stopped 'before 5'.

All local values and the $p c$.

Current pc (assumption).

Return to execution state 1.

and try else path.

$v(I<0)$.

New value of $O$.

Before 5.

Resume execution and delete breakpoint.

Turn all tracing off.

Reset $p c$ to true.

true; display $z$;

Go true above anticipates question.

Invoke editor to change absolute.

Edit command to look at line 1 of file.

Change proc name.

go to end of file.

Well not quite. 


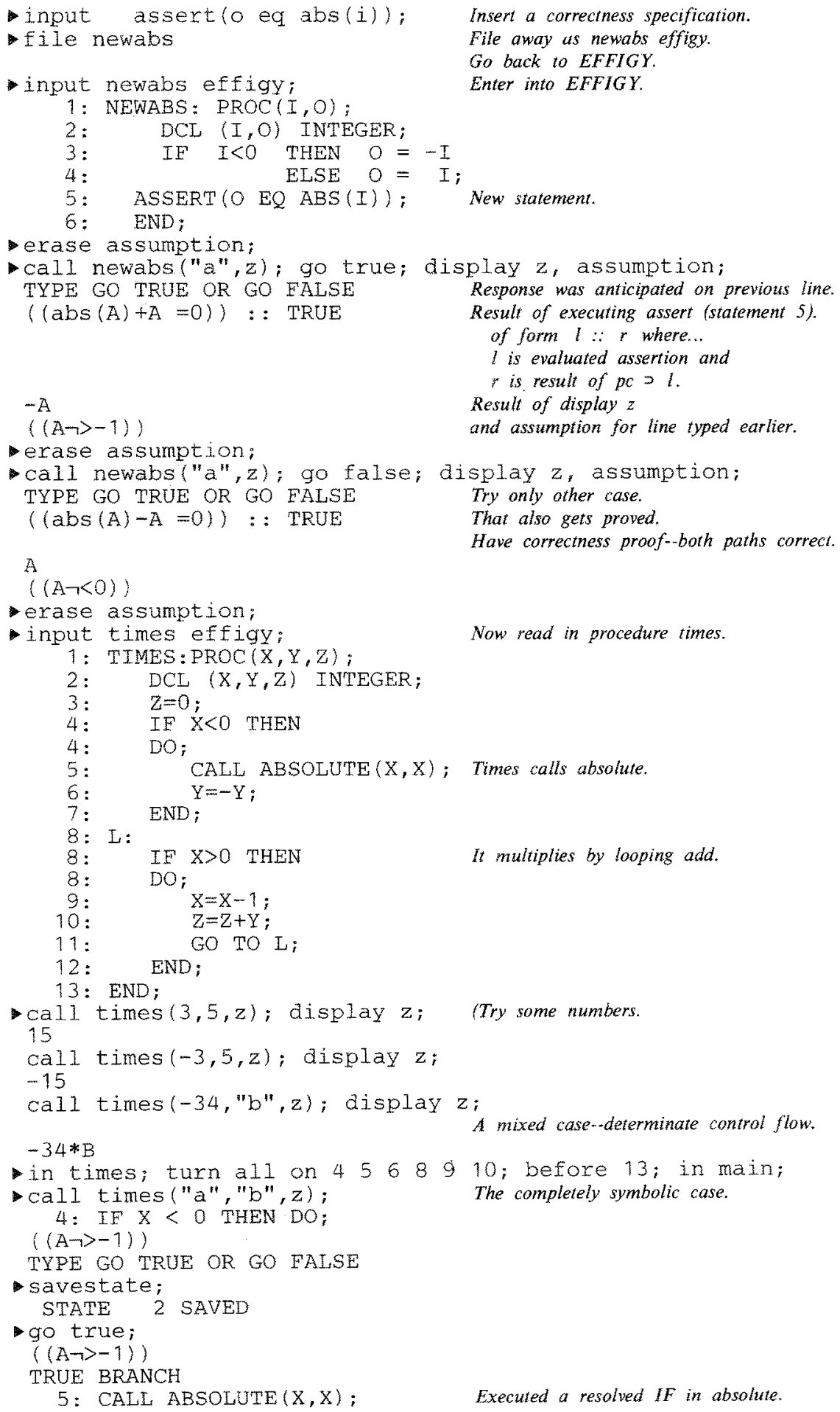


$\underset{Y=-B}{6: Y=-Y ;}$

8: L: IF $\mathrm{X}>0$ THEN DO;

$((A \neg>-1))$

TRUE BRANCH

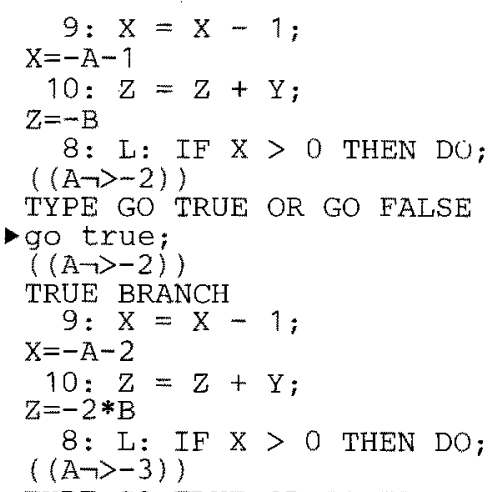




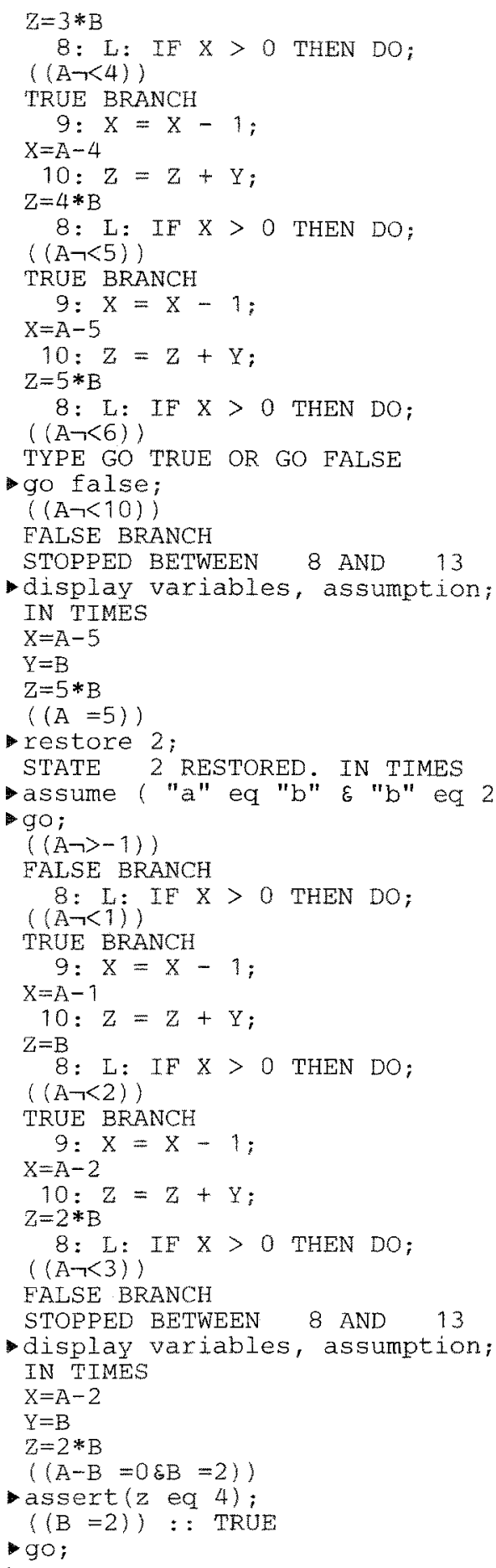

Unresolved when $X$ gets to $A-5$. Leave loop

Go back and try another case.

2) ; Indirectly assume $A$ is 2.

Does that assume resolve the if?

Yes it does.

This one resolved 100.

Result still in symbolic terms.

Does it know $Z$ is really 4 .

Yes.

Go on out of times.

Response to previous mull line. 


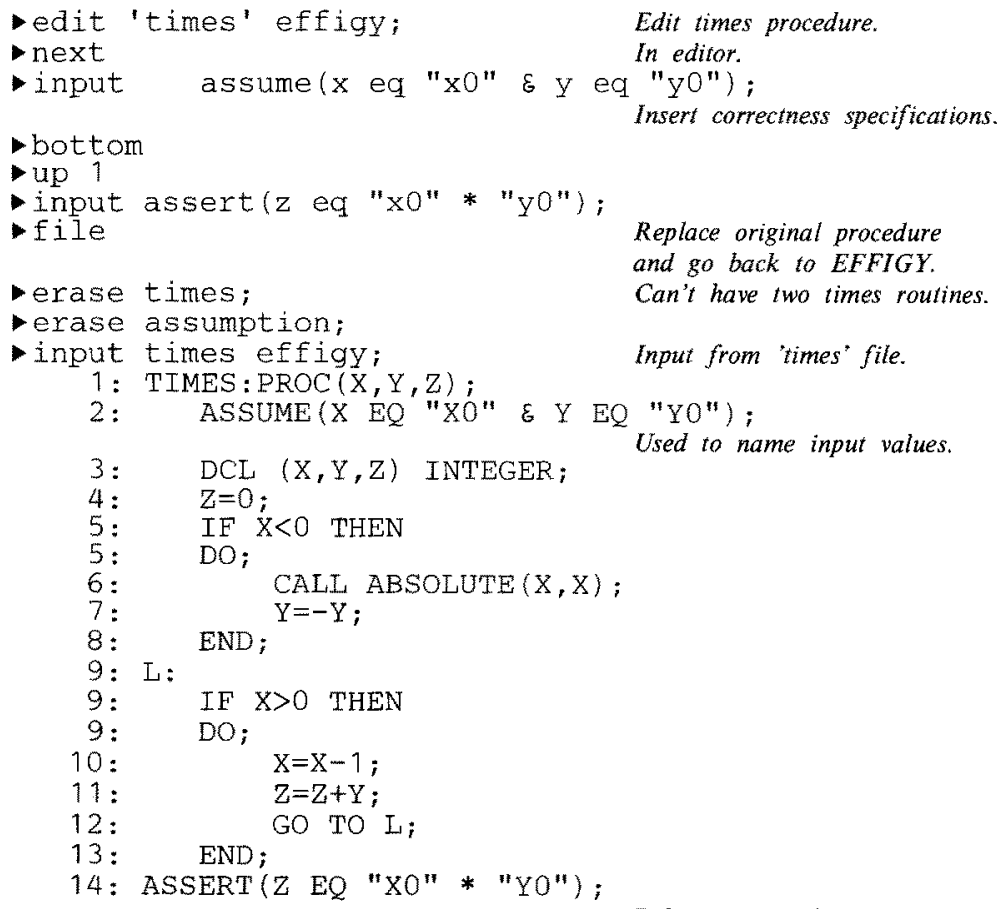

Now must decide 5 or 6.

Pick 6. 
$((A \neg<7))$

EALSE BRANCH

14: ASSERT ( $Z$ EQ XO * YO);

$((6 * \mathrm{~B}-\mathrm{XO} * Y \mathrm{O}=0)):$ :TRUE

- display assumption;

$((A=6 \& A-X O=0 \& B-Y O=0))$

- display variables;

IN MAIN

ABSOLUTE $=\mathrm{PROC}$

$Z=6 * B$

NEWABS $=$ PROC

TIMES=PROC

- quit
Known not $>6$.

Results check by assert-- O.K.

What is the pc?

Relates the symbolic inputs to the

names given to inputs by assume in proc.

MAIN has variables and values too.

Value 'PROC' means it is a procedure.

Leave EFFIGY system. 